\title{
Epifisiolistesis femoral proximal
}

\section{Slipped capital femoral epiphysis}

Alfonso Migoya-Nuño, ${ }^{1}$ José Antonio Delgado-Pérez, ${ }^{2}$ Óscar Daniel Isunza-Alonso, ${ }^{2}$ José Pablo Unda-Haro ${ }^{3}$

\section{ANTECEDENTES}

La epifisiolistesis femoral proximal es el desplazamiento de la epífisis sobre la metáfisis femoral proximal en la zona hipertrófica de la fisis o cartílago de crecimiento. ${ }^{1-4}$ Es la alteración de la cadera más común en los adolescentes, con incidencia global estimada en 1 de cada 1000 a 1 de cada 10,000 pacientes; ${ }^{5,6}$ el principal factor de riesgo es la obesidad, ${ }^{7}$ que aumenta en 1-650 casos para obesidad y 1-450 para obesidad severa. ${ }^{8}$ El $60 \%$ de los pacientes afectados se encuentra por arriba del percentil 90 para el género y la edad; sin embargo, la enfermedad es más frecuente en hombres que en mujeres (1.5:1) y el lado izquierdo suele ser el más afectado. La edad media para los hombres es de 13.5 años (cuando no han alcanzado el estadio IV de Tanner) y para las mujeres de 12 años, sin menarquia. Esta alteración es excepcional en pacientes menores de 10 y mayores de 16 años. ${ }^{6}$ Otros factores de riesgo incluyen: hipotiroidismo, deficiencia de la hormona de crecimiento, antecedente de radiación y trastornos genéticos (trisomía 21).9-12

\section{Manifestación clínica}

La epifisiolistesis femoral proximal puede aparecer en forma aguda o crónica. La primera se manifiesta en adolescentes con obesidad, quienes después de realizar un movimiento de abducción y rotación de cadera sufren dolor intenso en la región inguinal, que les incapacita para el apoyo y la marcha, motivo por el que suelen acudir al servicio de urgencias. En la forma crónica, los pacientes refieren dolor en la rodilla, con irradiación a la superficie anteromedial del tercio medio y distal del muslo; el inicio es progresivo, de intensidad variable, con episodios de exacerbación que cede con el reposo o consumo de analgésicos, y se acompaña de marcha claudicante, con cadera en rotación externa y limitación para la flexión de la cadera y la rodilla; ${ }^{1}$ en estos casos, el diagnóstico suele retrasarse, en promedio, 16 semanas. ${ }^{13}$

\footnotetext{
${ }^{1}$ Ortopedista pediatra, Hospital Ángeles Pedregal, Ciudad de México y Hospital Ángeles Puebla, Puebla.

${ }_{2}^{2}$ Adscrito al servicio de Ortopedia pediátrica, Instituto Nacional de Pediatría, Ciudad de México.

${ }^{3}$ Pediatra, Grupo Médico Pediátrico, Ciudad de México.

Recibido: 2 de julio 2019

Aceptado: 12 de agosto 2019

Correspondencia

Alfonso Migoya-Nuño

alfonsomigoya@gmail.com

Este artículo debe citarse como

Migoya-Nuño A, Delgado-Pérez JA, Isunza-Alonso OD, Unda-Haro JP. Epifisiolistesis femoral proximal. Acta Pediatr Mex. 2109;40(5):295-98.
} 


\section{Diagnóstico}

El diagnóstico se establece, en parte, con los haIlazgos clínicos: obesidad, signo de Drennan (al flexionar la cadera se desvía en rotación externa), dolor en rodillas, muslos, ingles, superficie lateral y posterior de la cadera; marcha claudicante y limitación para el apoyo. Y se comprueba con radiografías simples en proyección AP de pelvis y Lowenstein (Figura 1), en las que se observan: ensanchamiento e irregularidad de la fisis, deslizamiento anterior y en rotación externa de la metáfisis en relación con la fisis; línea de Klein, signo de Trethowan y de blancura de Steel.

\section{Clasificación}

De acuerdo con el tiempo de evolución: la forma aguda es menor de 3 y la crónica mayor de 3 semanas de evolución; la crónica agudizada mayor de 3 semanas de evolución se exacerba de manera súbita incapacitando el apoyo. ${ }^{3}$ En cuanto a la manifestación clínica: se considera estable cuando el paciente es capaz de tener apoyo y deambular sin complicaciones, e inestable cuando no puede realizar bipedestación ni marcha. ${ }^{4}$ Por lo que a mediciones radiográficas respecta: la enfermedad grado I sugiere desplazamiento de $0-30 \%$, grado II $31-60 \%$ y grado III mayor de $60 \%$; de acuerdo con la medición del ángulo de Southwick, se clasifica en leve, moderado y severo cuando es menor de $30^{\circ}$, entre $31-60^{\circ}$, y de $60^{\circ}$, respectivamente. ${ }^{14}$ Figuras 2 y 3

\section{Diagnóstico diferencial}

El diagnóstico diferencial se establece con fractura de cadera y lesiones de Labrum en pacientes con manifestación aguda, y con Legg-CalvePerthes ${ }^{3}$ osteomielitis y secuelas de displasia del desarrollo de cadera en quienes padecen la forma crónica de la enfermedad.

\section{Tratamiento}

El tratamiento de elección consiste en intervención quirúrgica, mediante la fijación in situ de la epífisis femoral proximal, con la colocación de un tornillo canulado (Figura 3); ${ }^{15,16}$ preferentemente deben fijarse ambas caderas, porque en $60 \%$ de los casos ocurre el deslizamiento en la cadera contralateral, en promedio a los 18 meses de la intervención, y en $90 \%$ de los casos con alteración metabólica. ${ }^{17,18,3}$
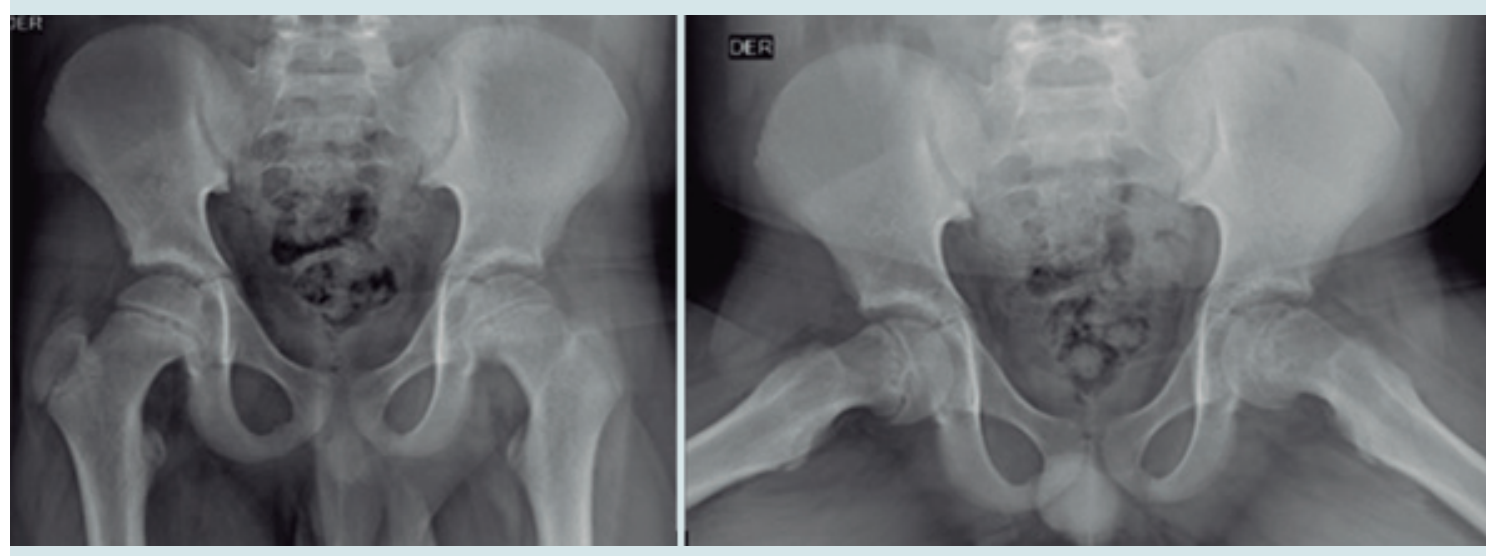

Figura 1. Radiografía simple. A) Proyección AP de pelvis y B) proyección de Lowenstein. 
Migoya-Nuño A, et al. Epifisiolistesis femoral proximal
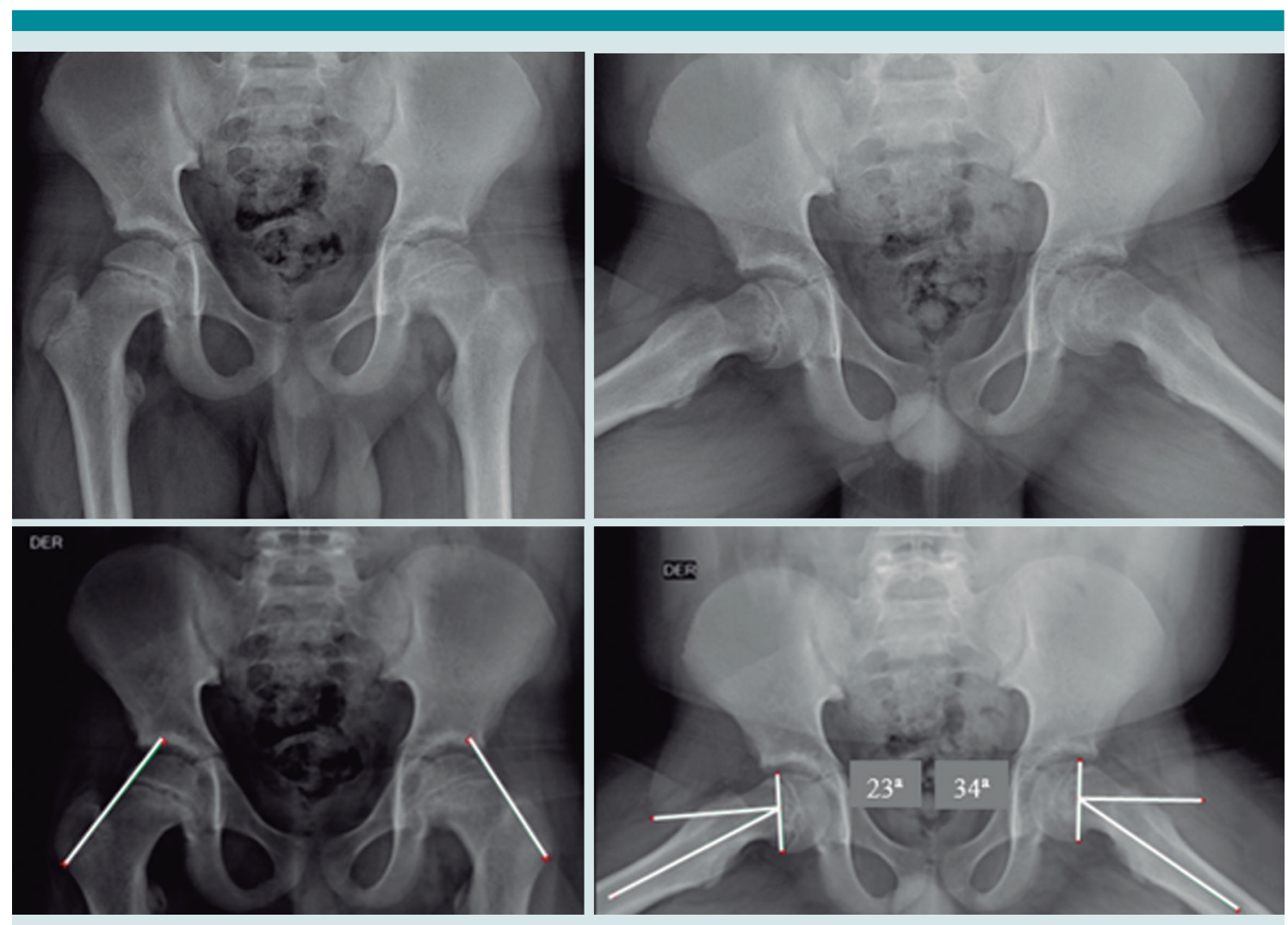

Figura 2. Epifisiolistesis femoral proximal bilateral. Medición radiográfica que muestra la cadera derecha, grado leve, ángulo de Southwick de $23^{\circ}$, cadera izquierda grado moderado y ángulo de Southwick $34^{\circ}$.
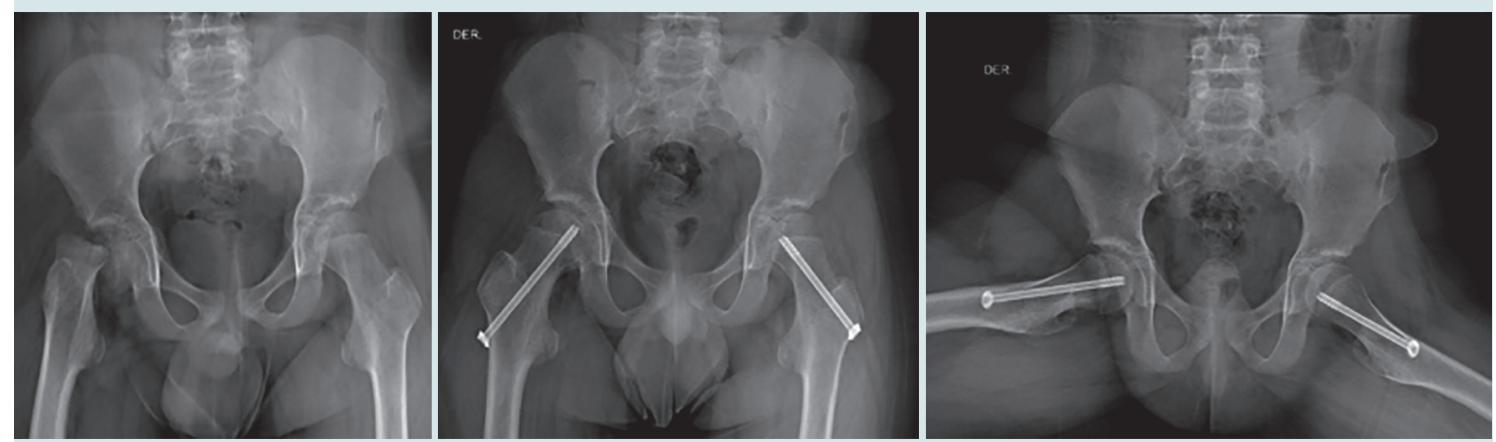

Figura 3. Epifisiolistesis femoral proximal derecha severa. Nótese que no es posible obtener la proyección de Soutwick, pues el paciente es incapaz de realizar la abducción de cadera. Imágenes posoperatorias que muestran la adecuada reducción, deslizamiento y fijación in situ de ambos lados de la cadera. 


\section{Intervención del especialista}

El envío al ortopedista pediatra debe efectuase tan pronto como se tenga la sospecha o se haya establecido el diagnóstico, pues el retraso en el tratamiento se asocia con mal pronóstico, debido al alto riesgo de osteonecrosis de la cabeza femoral. ${ }^{19,13}$

\section{SEGUIMIENTO}

El seguimiento debe ser anual, con el apoyo de radiografías simples y evaluación clínica, con la intención de evaluar los cambios morfológicos y alteraciones en la función, pues se ha observado que $30 \%$ de los pacientes manifiesta síntomas durante 15 años. ${ }^{20-22}$

\section{REFERENCIAS}

1. Georgiadis AG. Slipped Capi tal Femoral Epiphysis How to Evaluate with a Review and Update of Treatment. Pediatr Clin NA 2014;61(6):1119-35. DOI: http://dx.doi. org/10.1016/j.pcl.2014.08.001

2. Hellmich HJ, et al. Orthopäde. 2019; https://doi-org. pbidi.unam.mx:2443/10.1007/s00132-019-03743-4

3. Karkenny AJ, et al. Pediatric hip disorders: Slipped capital femoral epiphysis and legg-calvé-perthes disease.2018;39(9) :454-63. DOI: 10.1542/pir.2017-0197

4. Castillo $C$, et al. Slipped capital femoral epiphysis: a review for pediatricians. 2018;47(9):e377-e380. DOI: 10.3928/19382359-20180730-01

5. Lehmann $\mathrm{CL}$, et al. The Epidemiology of slipped capital femoral epiphysis: An update. 2006;26(3):286-90. DOI: 10.1097/01.bpo.0000217718.10728.70

6. Perry DC, et al. A nationwide cohort study of slipped capital femoral epiphysis. Arch Dis Child 2017;102:1132-1136 DOI: 10.1136/archdischild-2016-312328

7. Hoffmann S, et al. Orthopädische Probleme bei Adipositas im Kindes- und Jugendalter. Orthopedic problems in overweight and obese children. Klin Pediatr 2016;228(02):55-61. DOI: 10.1055/s-0035-1565214

8. Perry DC, et al. Childhood obesity and slipped capital femoral epiphysis. Pediatrics 2018;142(5):e2018106. DOI: https://doi.org/10.1542/peds.2018-1067
9. Kadowaki S, et al. Prepubertal onset of slipped capital femoral epiphysis associated with hypothyroidism. BMC Endocrine Disorders 2017;17(59)1-5. DOI: 10.1186/ s12902-017-0210-6

10. Bosch $P$, et al. Slipped capital femoral epiphysis in patients with down syndrome. 2004;24(3):271-7.

11. Vedi A, et al. Slipped capital femoral epiphyses after total body irradiation. Pediatr Blood Cancer 2014;61(6):24895. DOI: $10.1002 / p b c .24895$

12. Jagodzinski NA, et al. Prospective evaluation of a shortened regimen of treatment for acute osteomyelitis and septic arthritis in children. 2009;29(5):518-25.

13. Schur MD, et al. Continuing Delay in the Diagnosis of Slipped Capital Femoral Epiphysis. J Pediatr 2016;1-5. DOI: http://dx.doi.org/10.1016/j.jpeds.2016.06.029

14. Jones $\mathrm{CE}$, et al. Southwick angle measurements and SCFE slip severity classifications are affected by frog-lateral positioning. Skeletal Radiol 2018;47(1):1-6. DOI: 10.1007/ s00256-017-2761-z

15. Naseem $\mathrm{H}$, et al. Treatment of stable slipped capital femoral epiphysis : systematic review and exploratory patient level analysis. J Orthop Traumatol 2017;18(4):379-94. DOI: 10.1007/s10195-017-0469-4

16. Alshryda S, et al. Evidence based treatment for unstable slipped upper femoral epiphysis: Systematic review and exploratory patient level analysis. Surgeon 2018;16(1):4554. DOI: https://doi.org/10.1016/j.surge.2016.04.001

17. Bittersohl B, et al. Current concepts in management of slipped capital femoral epiphysis. Hip Int 2015;25(2):10414. DOI: 10.5301/hipint.5000189

18. Rodríguez RAL, et al. Aspectos epidemiológicos de la presentación bilateral de la epifisiolistesis femoral proximal. Rev Mex Ortop Ped 2007;1(9):5-9. https://www.medigraphic.com/cgi-bin/new/resumen.cgi?IDARTICULO=13071

19. Hosseinzadeh $P$, et al. Delay in the Diagnosis of Stable Slipped Capital Femoral Epiphysis. J Pediatr Orthop 2017;37(1):2015-8. DOI: 10.1097/ BPO.0000000000000665

20. Sucato DJ. Approach to the Hip for SCFE: The North American Perspective. 2018;38(6):5-12. DOI: 10.1097/ BPO.0000000000001183

21. Wright J, et al. Slipped Capital Femoral Epiphysis: The European Perspective. J Pediatr Orthop 2018;38(6):1-4. DOI: 10.1097/BPO.0000000000001161

22. Lang $P$, et al. The outcome of in-situ fixation of unstable slipped capital femoral epiphysis. J Pediatr Orthop 2019;16. DOI: $10.1097 /$ BPB. 0000000000000596 